\title{
Acetyl CoA Carboxylase Shares Control of Fatty Acid Synthesis with Fatty Acid Synthase in Bovine Mammary Homogenate
}

\author{
T. C. Wright, ${ }^{\star}$ J. P. Cant, ${ }^{\star}$ J. T. Brenna,† and B. W. McBride ${ }^{\star 1}$ \\ *Department of Animal and Poultry Science, University of Guelph, Guelph, ON, Canada N1G 2W1 \\ †Division of Nutritional Sciences, Cornell University, Ithaca, NY 14853
}

\begin{abstract}
The objectives of this research were to determine the flux control coefficients for acetyl CoA carboxylase and fatty acid synthase using an in vitro preparation of bovine mammary homogenate. For an enzyme to be considered rate limiting with the use of metabolic control analysis, its control coefficient would be equal to unity. The hypothesis for this experiment was that the control coefficient for acetyl CoA carboxylase was not equal to unity, and that this enzyme was not, therefore, the rate-limiting step. Mammary tissue was isolated from lactating Holstein cows at slaughter and frozen in liquid nitrogen. Tissue was ground, homogenized, and centrifuged to obtain a postmitochondrial supernatant for use in in vitro incubations containing labeled acetate. Specific inhibitors for acetyl CoA carboxylase and fatty acid synthase were used to fractionally inhibit de novo synthesis for the calculation of flux control coefficients. The composition of fatty acids synthesized in the absence of enzyme inhibitors was similar to the composition of fatty acids in the presence of inhibitors. Calculations following avidin inhibition of acetyl CoA carboxylase determined the flux control coefficient was $0.63 \pm 0.15$, which means that $63 \%$ of the control of fatty acid synthesis is exerted by acetyl CoA carboxylase. The remaining control (37\%) was from fatty acid synthase, which indicates a significant degree of control over the flux of acetate in de novo synthesis resides with this enzyme. The rate-limiting status ascribed to acetyl CoA carboxylase was not supported, because the flux control coefficient was less than unity. Metabolic control analysis, through its use of pathway product measurements, allows for potential interactions in the pathway such as feedback inhibition contribution to the flux control coefficients, which would not otherwise be considered in studies measuring enzyme kinetics with purified enzymes.
\end{abstract}

Key words: acetyl CoA carboxylase, fatty acid, fatty acid synthase, metabolic control analysis

Received November 6, 2005.

Accepted March 1, 2006.

${ }^{1}$ Corresponding author: bmcbride@uoguelph.ca

\section{INTRODUCTION}

In recent years, researchers have attempted to improve milk fat composition by dietary means such as feeding docosahexaenoic acid to introduce it into milk and by using feeding strategies that enhance the content of conjugated linoleic acids, which are naturally present in bovine milk (Jensen, 2002). However, the biosynthesis of fatty acids (FA), and milk fat itself, in the mammary glands is regulated by environmental and genetic factors (Barber et al., 1997) and approaches to manipulating milk fat by nutritional, genetic, or metabolic engineering will require a detailed understanding of the interactions between the components of this biosynthetic pathway.

Manipulations of milk fat composition inevitably alter the rates of some of the underlying biological processes that support de novo synthesis of FA; it is the regulation of processes such as substrate transport and enzyme activity or abundance that determines the final FA composition of milk. Fatty acids expressed in bovine milk are either synthesized de novo in the mammary glands from acetate and BHBA, or are absorbed from the bloodstream (Palmquist et al., 1993). Approximately half of palmitic acid and all of the longer chain length FA, are absorbed from blood, whereas short- and medium-chain FA and half of palmitic acid are synthesized within mammary epithelial cells.

De novo FA synthesis requires the coordinated functioning of numerous biological systems from the delivery of substrates to the mammary epithelial cells to the completion of the newly synthesized FA, to, finally, the formation of milk fat globules. Acetyl-CoA carboxylase (ACC; EC 6.4.1.2) is commonly described in the literature as the rate-limiting enzyme in the synthesis of de novo FA (Molenaar et al., 2003), although Volpe and Vagelos (1976) noted that the evidence from in vivo studies to support this position was not conclusive. Hardie (1989) suggested that the degree to which ACC could be considered rate limiting would depend on tissue type and the precursors available and noted that rate limiting would be more correctly defined in terms of 'control strength,' a forerunner to the termi- 
nology of control coefficient, used in metabolic control analysis (MCA). Acetyl-CoA carboxylase is the enzyme responsible for the formation of malonyl-CoA, the intermediary substrate necessary for chain elongation by the multifunctional enzyme FA synthase (FAS; EC 2.3.1.85; Smith, 1994).

Metabolic control analysis has been reviewed by Fell (1992, 1997). The MCA approach assumes a sharing or distribution of control among the individual elements of a biological pathway. Interactions within metabolic pathways, either through shared substrates or intermediates or from higher levels of biochemical organization, make pathways resistant to the redirection of flux from external modulation. The alternative concept of single control points, often described as ratelimiting or pace-maker steps, is replaced by the determination of control coefficients that represent the proportion of control exerted by each individual step. Plant biologists have already examined FA synthesis using the MCA approach (Page et al., 1994), and reported a flux control coefficient of 0.54 , or $54 \%$, for ACC. A 50-fold overexpression of the gene for ACC in Escherichia coli resulted in only a 6 -fold increase in FA synthesis (Davis et al., 2000), which indicates that a degree of control must be exerted by pathway constituents downstream from ACC. A complete examination of control coefficients for all steps of de novo FA synthesis in the mammary glands would require numerous experiments. The objective of this experiment was to determine the control coefficients for ACC and FAS in a defined in vitro system to test the hypothesis that ACC is rate-limiting for FA synthesis, and therefore has a control coefficient equal to unity.

\section{MATERIALS AND METHODS}

\section{Sample Collection}

Fresh mammary tissue was collected over 1 yr from 17 lactating Holstein cows immediately after slaughter in the abattoir of the Department of Animal and Poultry Science, University of Guelph. Cows were 245 $\pm 91 \mathrm{DIM}$ (mean $\pm \mathrm{SD}$ ) and parity of $3.2 \pm 1.4$. The University's Animal Care Committee, following the guidelines of the Canadian Council on Animal Care, approved animal use for this project. Cows were selected if their milk production equaled or exceeded 15 $\mathrm{kg} / \mathrm{d}$. Mammary samples were obtained from healthy mammary quarters after the gland was dissected following exsanguination. Sample collection and preparation followed the procedures of Wright et al. (2002). Briefly, approximately 0.5-g samples were isolated from the tissue, rinsed in saline, wrapped in foil, and snap frozen in liquid nitrogen. Tissue samples were subsequently processed into a fine powder under liquid nitrogen, and aliquots of prepared tissue were stored by cow in individual cryogenic tubes at $-70^{\circ} \mathrm{C}$. Frozen tissue was used within $30 \mathrm{~d}$ of initial freezing to ensure that tissue enzyme activity was maintained. A postmitochondrial supernatant from the tissue of each cow was obtained for use in subsequent in vitro incubations. Incubations for control coefficient calculations were performed in duplicate using 6 cows for avidin inhibition and 7 cows for pyridoxal 5-phosphate inhibition.

\section{In Vitro Incubations}

Incubations were conducted based on the methods of Wright et al. (2002), and chemicals were purchased from Sigma-Aldrich (Oakville, ON, Canada) except where noted. Concentrations in the final incubation solution ( $3 \mathrm{~mL}$ ) were $80 \mathrm{~m} M$ Tris- $\mathrm{HCl}, 0.8 \mathrm{mM} \mathrm{MnCl}$, $20 \mathrm{~m} M$ sodium bicarbonate, $0.05 \mathrm{~m} M$ coenzyme A, 1.7 $\mathrm{m} M$ ATP, $10 \mathrm{~m} M$ sodium citrate, $0.5 \mathrm{~m} M$ glucose 6 -P, $1.7 \mathrm{~m} M$ sodium acetate, $4.2 \mathrm{~m} M$ reduced glutathione, $0.05 \mathrm{~m} M$ NADPH, $20 \mathrm{mg} / \mathrm{mL}$ FA-free BSA, $5.0 \mu \mathrm{L}$ of mammalian protease inhibitor (P-8340), and 1 to 5 $\mathrm{mg}$ of homogenate protein. Vernon and Taylor (1986) noted that pyruvate carboxylase (EC 2.7.1.40) activity can contribute to assays intended to determine ACC activity based on ${ }^{14} \mathrm{C}$ incorporation from $\mathrm{H}^{14} \mathrm{CO}_{3}$ into acid-stable products when using homogenate preparations. Pyruvate, the substrate for pyruvate carboxylase activity was removed from the present assay by desalting the supernatant preparation using a Nanosep centrifugal concentrator with a $10-\mathrm{kDa}$ molecular weight cut-off filter (Northborough, MA) according to the manufacturer's directions.

The assay for ACC activity was adapted from the methods of Ingle et al. (1973) and Vernon and Taylor (1986) in which the incorporation of ${ }^{14} \mathrm{C}$ from $\mathrm{H}^{14} \mathrm{CO}_{3}$ into acid-stable products (e.g., malonyl-CoA) was measured. Approximately $1 \mu \mathrm{Ci}$ of $\mathrm{H}^{14} \mathrm{CO}_{3}$ (sodium salt) was added to the $3-\mathrm{mL}$ incubation solutions in 20 mL glass scintillation vials, and sealed with rubber stoppers containing a polypropylene center well (Kimble Kontes, Mandel Scientific Co., Guelph, ON). After $10 \mathrm{~min}$ in a shaking water bath at $37^{\circ} \mathrm{C}$, reactions were stopped by injecting $200 \mu \mathrm{L}$ of $6 N \mathrm{HCl}$ through the rubber stopper into the incubation solution. Two hundred microliters of tissue solubilizer (Amersham Canada Ltd., Oakville, ON, Canada) was placed in the center well before the incubations started to trap radioactive $\mathrm{CO}_{2}$ released after the addition of $\mathrm{HCl}$. Scintillation vials were removed from the water bath and transferred to a $60^{\circ} \mathrm{C}$ dry bath in a fume hood. Rubber stoppers and the center wells were removed after $1 \mathrm{~h}$ and crushed dry ice was added to the scintillation vials 
to eliminate unreacted $\mathrm{H}^{14} \mathrm{CO}_{3}$ (Ingle et al., 1973). A 1 -mL sample of the incubation solution from the scintillation vial was transferred to a clean scintillation vial and $10-\mathrm{mL}$ of universal scintillation cocktail (Formula 989, NEN Research, Boston, MA) was added. Scintillation spectroscopy was conducted in a Beckman LS6000SC scintillation counter (Fullerton, CA). Blank incubations were conducted without protein or substrate, and approximately $1 \mu \mathrm{Ci}$ of $\mathrm{H}^{14} \mathrm{CO}_{3}$ was counted for specific activity calculations. Homogenate protein content was estimated using the bicinchoninic reaction with BSA as the standard, according to kit instructions (Pierce, Rockford, IL).

Assays of FA synthesis rate were conducted according to Wright et al. (2002) for 60 min using the same incubation conditions as above, except that no $\mathrm{H}^{14} \mathrm{CO}_{3}$ was included in the incubations. Instead, 1 $\mu \mathrm{Ci}$ of $\left[1-{ }^{14} \mathrm{C}\right]$ acetic acid (sodium salt) was included as the label, and synthesized FA were extracted and counted by liquid scintillation. Net acetate incorporation was calculated by subtracting blank values from incubation results, and dividing by the specific activity of $\left[1-{ }^{14} \mathrm{C}\right]$ acetic acid.

Inhibition of enzyme activity for calculation of control coefficients was accomplished with avidin for ACC and pyridoxal 5-phosphate for FAS (Wakil et al., 1983). From 0 to $10 \mu M$ avidin was added to incubation solutions described above after a brief activation period for the homogenate protein in the incubation solution.

Individual FA synthesized de novo in the homogenate were determined by analyzing incubations that used a stable isotope of acetate instead of the radioisotope. A preliminary set of incubations indicated that a 1:50 ratio of $\left[1,2-{ }^{13} \mathrm{C}\right]$ acetate (Cambridge Isotope Laboratories Inc., Andover, MA) with natural abundance acetate was sufficient to measure the incorporation of $\left[1,2-{ }^{13} \mathrm{C}\right]$ acetate into FA. Stable isotope incubations containing no inhibitors, 1 and $2 \mu M$ avidin, or pyridoxal 5 -phosphate were stopped by immersing in a salt and ice mixture, and immediately frozen at $-20^{\circ} \mathrm{C}$. Fatty acids were extracted from the incubations containing stable isotope by using 2 extractions of 1:2 chloroform:methanol (vol/vol), and transmethylated in the presence of boron trichloride. The purified FA methyl esters were disolved in hexane (Lipid Analytic Laboratories, Guelph, ON) before transport to Cornell University (Ithaca, NY). Methyl esters labeled with ${ }^{13} \mathrm{C}$ acetate were analyzed by gas chromatographycombustion isotope ratio mass spectrometry (GCCIRMS) according to the methods described by Su and Brenna (1998) and Huang et al. (2000).

\section{Data Analysis}

Metabolic control analysis was conducted using only the results obtained from the assays that measured the incorporation of radiolabeled substrate into total FA. Flux control coefficients $\left(\mathbf{C}^{\boldsymbol{J}}\right)$ for each enzyme $\left(\mathrm{E}_{\mathrm{i}}\right)$ representing the degree to which total FA synthesis was affected by either ACC or FAS inhibition were calculated according to Derr $(1985,1986)$ :

$$
C_{E_{i}}^{J}=\frac{1}{J}\left(\frac{\partial J}{\partial I}\right)_{I=0} \cdot-I_{\max }\left(1+\frac{S}{K_{S}}\right)
$$

where $\mathbf{J}$ is the uninhibited rate of FA synthesis, ( $\partial \mathrm{J} /$ $\partial \mathrm{I})_{\mathrm{I}=0}$ is the initial slope of the decline in FA synthesis at zero inhibition, $\mathrm{I}_{\max }$ is maximal inhibition value, $S$ is substrate concentration, and $K_{s}$ is the substrate constant.

The summation theorem (Fell, 1992) was used to calculate the remaining control coefficient as:

$$
C_{E_{2}}^{J}=1-C_{E_{1}}^{J} \text {. }
$$

The value of $(\partial \mathrm{J} / \partial \mathrm{I})_{\mathrm{I}=0}$ was obtained by first estimating inhibition constant $\mathrm{K}_{\mathrm{i}}$ from fits of the equation Inhibition $=\mathrm{I} /\left[\mathrm{I}+\mathrm{K}_{\mathrm{i}} \times\left(1+\mathrm{S} / \mathrm{K}_{\mathrm{S}}\right)\right]$ to plots of degree of inhibition of FA synthesis vs. inhibitor concentration (I) using the nonlinear regression procedure of SAS (SAS Institute, 2000). The value of $\mathrm{K}_{\mathrm{s}}$ was estimated from plots of ACC activity vs. acetate concentration utilizing the software Leonora (version 1.0), which was designed for analysis of enzyme kinetic data (CornishBowden, 1995); I MAX was determined by titration with inhibitor until less than $5 \%$ of uninhibited assay activity was detected.

Control coefficient results were tested for a difference from unity $\left(C^{J}=1.0\right)$ by Student's $t$-test using SAS (SAS, Institute, 2000). Data for the incorporation of the stable isotope of acetate into FA were analyzed as a completely randomized design using Proc GLM of SAS (SAS, Institute, 2000). Differences in the profiles of FA synthesized between treatments and control were detected using the PDIFF option after a significant $F$-statistic. Significance was taken as $P<0.05$.

\section{RESULTS AND DISCUSSION}

\section{Fatty Acid Profile}

The use of inhibitors or activators of enzyme activity in MCA requires that, for infinitesimal activity changes, there is no significant change in the products synthesized by the pathway. The pattern of FA produced by bovine FAS includes short-, medium-, and long-chain FA. The greatest incorporation of $\left[1,2-{ }^{13} \mathrm{C}\right]$ acetate for all incubations was into $\mathrm{C}_{14: 0}$ (Figure 1), with approximately 46 and $33 \%$ of that amount incorporated into $\mathrm{C}_{10: 0}$ and $\mathrm{C}_{12: 0}$, respectively, in the control 


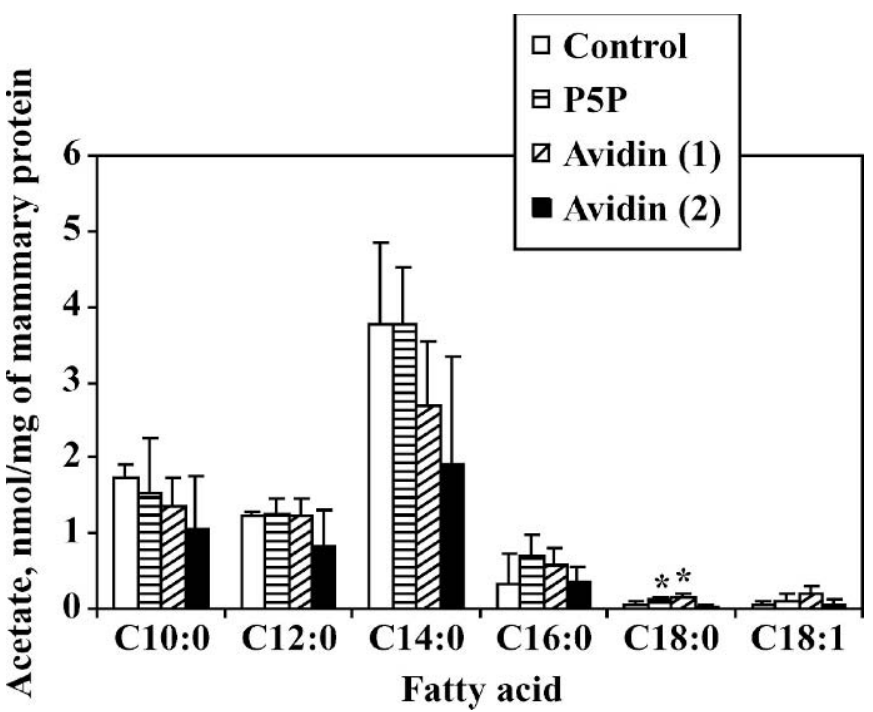

Figure 1. Acetate incorporation (mean $\pm \mathrm{SD}$ ) into fatty acids from control and inhibited incubations ( $\mathrm{P} 5 \mathrm{P}=$ pyridoxal 5 -phosphate, and avidin at 1 and $2 \mu M)$. * Denotes statistical difference from control $(P<0.05)$.

incubations. Incorporation of acetate into $\mathrm{C}_{16: 0}$ was less than $10 \%$ in control incubations, which contrasts with in vivo $\mathrm{FA}$ synthesis, in which $\mathrm{C}_{16: 0}$ is the primary de novo product (29.5\%; Palmquist et al., 1993). In ruminants, the presence of different FA chain lengths in milk results from a lack of specificity of the acyltransferase responsible for loading acetyl and malonyl substrates. The specificity of the acyltransferase in ruminants extends to short and medium chain lengths so that several chain lengths can in fact equilibrate between the CoA ester and enzyme-bound forms (Smith, 1994). This equilibration allows ruminant mammary glands to incorporate short and medium chain lengths into triacylglycerols because of the close association of FA synthesis and triacylglycerol synthesis. The disruption of the association between these 2 systems (i.e., the absence of a microsomal fraction in the present system) would be expected to result in differences between in vitro and in vivo FA profiles because of the differences in chain termination. In the present in vitro system, acetate is incorporated into free FA unlike the formation of triacylglycerols in vivo (Wright et al., 2002).

The only significant effect of the inhibitors was on $\mathrm{C}_{18: 0}$ synthesis by pyridoxal 5 -phosphate and the 1 $\mu M$ avidin incubation. Detectable quantities of labeled acetate were measured in $\mathrm{C}_{18: 0}$ and $\mathrm{C}_{18: 1}$, indicating elongation and desaturation by mechanisms external to the ACC-FAS synthesis pathway. Incorporation of trace amounts of labeled acetate into $\mathrm{C}_{18} \mathrm{FA}$ in homog- enate was reported by DeKay et al. (1976). The potential for a functional FA chain-lengthening elongase system remaining after the preparation of the mammary tissue was not discounted in the present experiment. The data in Figure 1 demonstrate the similarity of FA patterns in control and inhibited incubations; in particular, there were no differences in those fatty acids measured with a chain length of $\mathrm{C}_{16}$ or shorter.

In vitro mammary preparations have previously been used to investigate FA synthesis. DeKay et al. (1976), using a cytosolic preparation of bovine mammary tissue, reported incorporation of labeled acetate into $\mathrm{C}_{4: 0}, \mathrm{C}_{6: 0}$, and $\mathrm{C}_{8: 0}$ of 15,10 , and $8 \%$, respectively, and equal incorporation into $\mathrm{C}_{14: 0}$ and $\mathrm{C}_{16: 0}$ pools. The similar incorporation into $\mathrm{C}_{14: 0}$ and $\mathrm{C}_{16: 0}$ was primarily a result of reduced incorporation into $\mathrm{C}_{16: 0}$ compared with expectations from in vivo measures. Conditions used herein for the GCC-IRMS did not permit the separation of short-chain FA. Hansen and Knudsen (1987) reported the profile of de novo FA synthesized by bovine mammary epithelial cells that incorporated [1$\left.{ }^{14} \mathrm{C}\right]$ acetate into several lipid classes including free FA and triacylglycerols. As in the present experiments, the greatest incorporation of acetate was into $\mathrm{C}_{14: 0}$. These data indicate that for in vitro models of bovine mammary FA synthesis, there is a change in the FA profile produced compared with in vivo FA synthesis, likely due to the differences in the intracellular associations between FA synthesis and triacylglycerol synthesis machinery. The similarity in FA profiles between treatments confirms that MCA can be applied to the ACC and FAS synthesis fluxes by the inhibitors used herein. The MCA analysis accounted for all FA chain lengths synthesized by using the data obtained from the incorporation and detection of radiolabeled substrate into total FA.

\section{Flux Control Coefficients}

Inhibition of acetate incorporation into FA by avidin is shown in Figure 2. The data from Figure 2 were used to calculate the initial slope of the normalized flux as inhibitor concentration approached zero. The value of $\mathrm{K}_{\mathrm{s}}$ obtained from the radiolabeled bicarbonate incubations was $3.51 \times 10^{-6}$, and the maximal inhibitor concentration was $8.7 \times 10^{-3} \mathrm{mM}$. The initial slope of the avidin inhibition curve as the inhibitor concentration approached the limit of zero was calculated as $-0.15 \pm 0.04$

Less than $3 \%$ activity was detected ( $\mathrm{n}=4$ cows) at the inhibitor maximum value for acetate. Calculations yielded a flux control coefficient of $0.63 \pm 0.15$ using equation 1. Application of the summation equation (equation 2) indicates that the flux control coefficient 


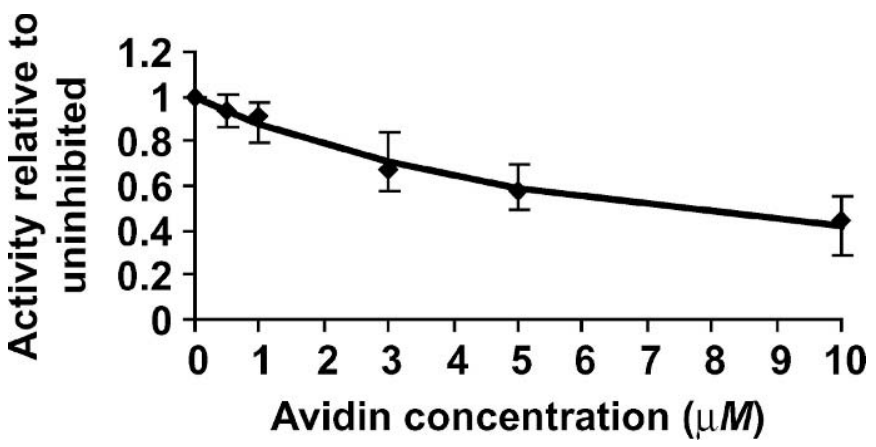

Figure 2. Inhibition of de novo fatty acid synthesis by avidin; each point represents the mean $( \pm \mathrm{SE})$ of 6 observations of fatty acid synthesis rate. The solid line shows the least squares fit of the equation: Inhibition $=\mathrm{I} /\left(\mathrm{I}+\mathrm{K}_{\mathrm{i}} \times\left(1+\mathrm{S} / \mathrm{K}_{\mathrm{s}}\right)\right]$.

for FAS was 0.37 , which means that $37 \%$ of the control of de novo synthesis of FA in the in vitro system resided with FAS. The use of labeled acetate for the measurement of de novo FA synthesis herein means that the apparent control coefficient for ACC is an aggregate determination for ACC and acetate CoA ligase (EC 6.2.1.1). Acetate CoA ligase is the enzyme that catalyzes the formation of acetyl-CoA from acetate and $\mathrm{CoA}$ in a reaction that consumes $2 \mathrm{~mol}$ of high-energy phosphate bonds/mol of acetyl-CoA formed.

Data from the experiments using pyridoxal 5-phosphate to inhibit FAS indicated that the control coefficient for FAS was 0.24; and, according to the summation theorem (equation 2), the control coefficient for ACC was 0.76. However, the drawback to the use of an inhibitor for the second reaction in a pathway is that, whereas in the first reaction, substrate concentration is experimentally set, that is not the case for downstream reactions. The reduced activity of FAS could increase the concentration of the intermediate substrate, malonyl-CoA, because it would be utilized more slowly by the inhibited FAS, and cause underestimation of the control coefficient. Therefore, although there is some consistency to the results of the 2 approaches for calculating a control coefficient, the results of the avidin inhibition correctly satisfy the requirements of MCA that concentrations remain constant. It should be noted that, in either case, the control coefficient calculated for ACC was significantly different from unity.

In general, the use of subcellular preparations is less desirable as an experimental model for MCA than experiments that use whole cells or organelles such as mitochondria (Fell, 1997). The cell membrane disruption that occurs when homogenates are prepared eliminates potential control from transporters. Torres et al. (1986) studied glycolytic activity in rat liver ho- mogenates using an enzyme addition technique to increase pathway flux. Despite the constraints of their homogenate model (such as a more dilute medium than the cytosol, and truncation of the pathway), their findings were consistent with subsequent investigations using whole-cell models (Fell, 1997). Use of homogenate models provides valuable insight into in vivo situations.

Inhibition of ACC by avidin was studied in avian liver (Wakil et al., 1958). In their experiment, incremental reductions in FA synthesis were achieved with corresponding increments of avidin. The lowest reduction in FA synthesis was approximately $20 \%$; however, there was no report of lower inhibitor concentration use. DeKay et al. (1976) and Gnoni and Muci (1990) reported effects of avidin inhibition of FA synthesis in bovine mammary homogenate and eel-liver cytosol, respectively. DeKay et al. (1976) reported $>90 \%$ inhibition of FA synthesis when their mammary preparation was preincubated with avidin before addition to the incubation medium. This preincubation approach differed from the approach used herein, and the resulting pattern of FA synthesized in their experiment shifted to a pattern dominated by butyric acid with the avidin preincubation treatment. Gnoni and Muci (1990) utilizing an avidin preincubation treatment reported results that are in agreement with others (DeKay et al., 1976). The avidin inhibition in the present system exhibited reduced sensitivity compared with the preincubation technique, but functioned similarly to the effects reported for avian ACC by Wakil et al. (1958).

\section{Role of ACC in Milk Fat Synthesis}

Our estimates of control coefficients indicate that $\mathrm{ACC}$ is not the rate-limiting step in de novo synthesis of FA in vitro. The term "rate-limiting" ascribed to ACC has been used for a long time and remains in use currently (Molenaar et al., 2003). A control coefficient less than 1.0 does not diminish the metabolic importance of ACC in the pathway for de novo FA synthesis, and a control coefficient of 0.63 represents a substantial degree of control over the complete pathway flux.

Davis et al. (2000) tested the effects of ACC overexpression in $E$. coli to examine the rate-controlling nature of this enzyme. There are differences between bacterial and mammalian ACC, but their findings represented the first direct approach to examining ACC control of FA synthesis in vivo. In their experiment, the potential for feedback from accumulating FA products was removed by incorporating a mutant thioesterase that directed overproduced FA into the culture medium, which served as a convenient metabolic sink. Overexpression resulted in an approximately 50-fold 
increase in ACC activity, and a 100-fold increase in intracellular malonyl-CoA concentration, but only a 6fold increase in the rate of free FA synthesis, which indicated that subsequent pathway steps were exerting a degree of control over total pathway flux. Thus, ACC exerted strong effects on flux when activated (or inhibited), but, in the absence of complete control, limitations to the increases in flux anticipated from enzyme activity changes were apparent from the increased malonyl-CoA level.

Milk fat depression was associated with decreased activity of ACC and FAS in mammary tissue (Piperova et al., 2000). Similarly, milk fat depression induced by feeding a high concentrate/low forage diet was associated with significant reductions in mammary mRNA abundances for ACC, FAS, stearoyl-CoA desaturase, lipoprotein lipase, FA binding protein, fatty acyl CoA ligase, glycerol phosphate acyl transferase, and acylglycerol phosphate acyl transferase (Peterson et al., 2003). Biological regulation that causes a cell to reduce many enzymes in a metabolic pathway is likely an appropriate and efficient response for the cell to balance intermediate metabolite concentrations with reduced energy expenditure. It is a further indication that control is distributed over many of the components of metabolic pathways. Bauman and Griinari (2003) suggested further candidates that may provide control points in milk fat synthesis, namely peroxisome proliferator-activated receptors and sterol regulatory element binding proteins. Examining the transport of FA and substrates for de novo FA synthesis into mammary epithelial cells and components of the mechanism by which milk fat globules are secreted would likely reveal additional control points for FA synthesis.

\section{CONCLUSIONS}

The existence of a high flux control coefficient for ACC in corn and barley (Page et al., 1994) and the indication of similar importance in $E$. coli (Davis et al., 2000) may be indicative of the importance of ACC to the control of mammalian FA synthesis. The high flux control coefficient in the in vitro model used herein indicates the significance of ACC to the control over de novo FA synthesis. However, shared control with FAS means that ACC should not be described in terms of being a rate-limiting enzyme. The complexities of in vivo FA synthesis and additional candidates for control indicate that ACC is not the rate-limiting step for milk FA synthesis.

\section{ACKNOWLEDGMENTS}

The financial support of NSERC (to B.W.M.), the Ontario Ministry of Agriculture and Food and NIH grant GM49209 (to J.T.B) for this work was appreciated. The assistance of Peter Lawrence for the GCCIRMS analysis at Cornell University, Glenn Snider at the University of Guelph meat laboratory, and Angela Fairfield at the Elora Dairy Cattle Research Centre was especially helpful. A portion of this work was presented at the Xth International Symposium on Ruminant Physiology, Copenhagen, Denmark, 2004.

\section{REFERENCES}

Barber, M. C., R. A. Clegg, M. T. Travers, and R. G. Vernon. 1997. Lipid metabolism in the lactating mammary gland. Biochim. Biophys. Acta 1347:101-126.

Bauman, D. E., and J. M. Griinari. 2003. Nutritional regulation of milk fat synthesis. Annu. Rev. Nutr. 23:203-227.

Cornish-Bowden, A. 1995. Analysis of enzyme kinetic data. Oxford University Press, Oxford, UK.

Davis, M. S., J. Solbiati, and J. E. Cronan. 2000. Overproduction of acetyl-CoA carboxylase activity increases the rate of fatty acid biosynthesis in Escherichia coli. J. Biol. Chem. 275:2859328598.

DeKay, D. E., D. E. Bauman, and C. L. Davis. 1976. Characterization of fatty acid synthesis by cow mammary subcellular fractions. J. Dairy Sci. 59:1513-1517.

Derr, R. F. 1985. Modern metabolic control theory 1. Fundamental theorems. Biochem. Arch. 1:239-247.

Derr, R. F. 1986. Modern metabolic control theory. 2. Determination of flux-control coefficients. Biochem. Arch. 2:31-44.

Fell, D. A. 1992. Metabolic control analysis: A survey of its theoretical and experimental development. Biochem. J. 286:313-330.

Fell, D. A. 1997. Understanding the control of metabolism. Portland Press Ltd., London, UK.

Gnoni, G. V., and M. R. Muci. 1990. De novo fatty acid synthesis in eel-liver cytosol. Comp. Biochem. Physiol. Part B. 95:153-158.

Hansen, H. O., and J. Knudsen. 1987. Effect of exogenous longchain fatty acids on lipid biosynthesis in dispersed ruminant mammary gland epithelial cells: Esterification of long-chain exogenous fatty acids. J. Dairy Sci. 70:1344-1349.

Hardie, D. G. 1989. Regulation of fatty acid synthesis via phosphorylation of acetyl-CoA carboxylase. Prog. Lipid Res. 28:117-146.

Huang, M. C., S. Muddana, E. N. Horowitz, C. C. McCormick, J. P. Infante, and J. T. Brenna. 2000. High-precision isotope ratio mass spectrometry and stable isotope precursors for tracer studies in cell culture. Anal. Biochem. 287:80-86.

Ingle, D. L., D. E. Bauman, R. W. Mellenberger, and D. E. Johnson. 1973. Lipogenesis in the ruminant: Effect of fasting and refeeding on fatty acid synthesis and enzymatic activity of sheep adipose tissue. J. Nutr. 103:1479-1488.

Jensen, R. G. 2002. The composition of bovine milk lipids: January 1995 to December 2000. J. Dairy Sci. 85:295-350.

Molenaar, A., J. Mao, K. Oden, and H. M. Seyfert. 2003. All three promoters of the acetyl-coenzyme A-carboxylase ALPA-encoding gene are expressed in mammary epithelial cells of ruminants. J. Histochem. Cytochem. 51:1073-1081.

Page, R. A., S. Okada, and J. L. Harwood. 1994. Acetyl-CoA carboxylase exerts strong flux control over lipid synthesis in plants. Biochim. Biophys. Acta 1210:369-372.

Palmquist, D. L., A. D. Beaulieu, and D. M. Barbano. 1993. Feed and animal factors influencing milk fat composition. J. Dairy Sci. 76:1753-1771.

Peterson, D. G., E. A. Matitashvili, and D. E. Bauman. 2003. Dietinduced milk fat depression in dairy cows results in increased trans-10, cis-12 CLA in milk fat and coordinate suppression of mRNA abundance for mammary enzymes involved in milk fat synthesis. J. Nutr. 133:3098-3102.

Piperova, L. S., B. B. Teter, I. Bruckental, J. Sampugna, S. E. Mills, M. P. Yurawecz, J. Fritsche, K. Ku, and R. A. Erdman. 2000. 
Mammary lipogenic enzyme activity, trans fatty acids and conjugated linoleic acids are altered in lactating dairy cows fed a milk fat-depression diet. J. Nutr. 130:2568-2574.

SAS Institute. 2000. Software Release 8.1. SAS Institute, Inc., Cary, NC.

Smith, S. 1994. The animal fatty acid synthase: One gene, one polypeptide, seven enzymes. FASEB J. 8:1248-1259.

$\mathrm{Su}, \mathrm{H}$. M., and J. T. Brenna. 1998. Simultaneous measurement of desaturase activities using stable isotope tracers or a nontracer method. Anal. Biochem. 261:43-50.

Torres, N. V., F. Mateo, E. Melendez-Hevia, and H. Kacser. 1986. Kinetics of metabolic pathways: A system in vitro to study the control of flux. Biochem. J. 234:169-174.
Vernon, R. G., and E. Taylor. 1986. Acetyl-CoA carboxylase of sheep adipose tissue: Problems of the assay and adaptation during fetal development. J. Anim. Sci. 63:1119-1125.

Volpe, J. J., and P. R. Vagelos. 1976. Mechanisms and regulation of biosynthesis of saturated fatty acids. Physiol. Rev. 56:339-418.

Wakil, S. J., J. K. Stoops, and V. C. Joshi. 1983. Fatty acid synthesis and its regulation. Annu. Rev. Biochem. 52:537-579.

Wakil, S. J., E. B. Titchener, and D. M. Gibson. 1958. Evidence for the participation of biotin in the enzymic synthesis of fatty acids. Biochim. Biophys. Acta 29:225-226.

Wright, T. C., J. P. Cant, and B. W. McBride. 2002. Inhibition of fatty acid synthesis in bovine mammary homogenate by palmitic acid is not a detergent effect. J. Dairy Sci. 85:642-647. 